\title{
A note on the almost sure central limit theorems for the maxima of strongly dependent nonstationary Gaussian vector sequences
}

\section{Xiang Zeng and Qunying Wu*}

${ }^{*}$ Correspondence: wqy666@glut.edu.cn College of Science, Guilin University of Technology, Guilin, Guangxi 541004, P.R. China

\begin{abstract}
We prove some almost sure central limit theorems for the maxima of strongly dependent nonstationary Gaussian vector sequences under some mild conditions. The results extend the ASCLT to nonstationary Gaussian vector sequences and give substantial improvements for the weight sequence obtained by Lin et al. (Comput. Math. Appl. 62(2):635-640, 2011).
\end{abstract}

MSC: 60F15

Keywords: almost sure central limit theorem; strongly dependent nonstationary Gaussian vector sequences; weight sequence

\section{Introduction}

The almost sure central limit theorem (ASCLT) has served as a basis for a large group of investigations of fundamental significance both in the theory of probability and in its numerous applications to statistics, natural sciences, engineering, and economics. Its methods and results continue to have great influence on other fields of probability theory, mathematical statistics, and their applications. In recent decades, there has been much work on the ASCLT. Cheng et al. [2], Fahrner and Stadtmüller [3], and Berkes and Csáki [4] considered the ASCLT for the maximum of i.i.d. random variables. For more related work on ASCLT, see [5-13]. An influential work is Csáki and Gonchigdanzan [14], which proved the almost sure limit theorem for the maximum of stationary weakly dependent sequence. Furthermore, Lin [15] considered the theorem which ASCLT version of the theorem proved by Leadbetter et al. [16]. Chen et al. [17] extended [14] to the multivariate stationary case. Lin et al. [1] partially extended [14] to the case of strongly dependent nonstationary Gaussian sequences and obtained the following theorem.

Theorem A Let $\left\{\xi_{n}: n \geq 1\right\}$ be a sequence of nonstationary standard Gaussian random variables with covariances $r_{i j}$ satisfying $\left|r_{i j}-\frac{r}{\ln (j-i)}\right| \ln (j-i)(\ln \ln (j-i))^{1+\varepsilon}=O(1)$ for $r>0$. If

$$
a_{n}=(2 \ln n)^{1 / 2}, \quad b_{n}=(2 \ln n)^{1 / 2}-\frac{1}{2}(2 \ln n)^{-1 / 2}(\ln \ln n+\ln (4 \pi)),
$$

(0) 2016 Zeng and Wu. This article is distributed under the terms of the Creative Commons Attribution 4.0 International License (http://creativecommons.org/licenses/by/4.0/), which permits unrestricted use, distribution, and reproduction in any medium, provided you give appropriate credit to the original author(s) and the source, provide a link to the Creative Commons license, and indicate if changes were made. 
then

$$
\lim _{n \rightarrow \infty} \frac{1}{\ln n} \sum_{k=1}^{n} \frac{1}{k} I\left(a_{k}\left(M_{k}-b_{k}\right) \leq x\right)=\int_{-\infty}^{\infty} \exp \left(-\mathrm{e}^{-x-r+\sqrt{2 r} z}\right) \phi(z) \mathrm{d} z \quad \text { a.s. }
$$

where I denotes an indicator function and $\phi$ is the standard normal density function.

The purpose of this paper is to give substantial improvements for both weight sequence and the range of random variables of Theorem $\mathrm{A}$.

Throughout the paper, let $\left\{\xi_{i}=\left(\xi_{i}(1), \xi_{i}(2), \ldots, \xi_{i}(d)\right): i \geq 1\right\}$ be a standardized nonstationary Gaussian vector sequence with

$$
\begin{aligned}
& \mathbb{E} \boldsymbol{\xi}_{n}=\left(\mathbb{E} \xi_{n}(1), \mathbb{E} \xi_{n}(2), \ldots, \mathbb{E} \xi_{n}(d)\right)=(0,0, \ldots, 0), \\
& \operatorname{Var} \boldsymbol{\xi}_{n}=\left(\operatorname{Var} \xi_{n}(1), \operatorname{Var} \xi_{n}(2), \ldots, \operatorname{Var} \xi_{n}(d)\right)=(1,1, \ldots, 1), \\
& r_{i j}(p)=\operatorname{Cov}\left(\xi_{i}(p), \xi_{j}(p)\right), \\
& r_{i j}(p, q)=\operatorname{Cov}\left(\xi_{i}(p), \xi_{j}(q)\right), \quad \text { for } 1 \leq p \neq q \leq d .
\end{aligned}
$$

Let $\left\{\boldsymbol{\eta}_{i}=\left(\eta_{i}(1), \eta_{i}(2), \ldots, \eta_{i}(d)\right): i \geq 1\right\}$ be a $d$-dimensional vector sequence. For $i \geq 1$, we define

$$
\boldsymbol{\xi}_{i} \boldsymbol{\eta}_{i}=\left(\xi_{i}(1) \eta_{i}(1), \xi_{i}(2) \eta_{i}(2), \ldots, \xi_{i}(d) \eta_{i}(d)\right)
$$

Let $\mathbf{u}_{n i}=\left(u_{n i}(1), u_{n i}(2), \ldots, u_{n i}(d)\right)$ be a $d$-dimensional real vector, and $\mathbf{u}_{n i}>\mathbf{u}_{k i}$ means $u_{n i}(p)>u_{k i}(p)$ for $p=1,2, \ldots, d$. Suppose

$$
r_{i j}(p) \ln (j-i) \rightarrow r, \quad r_{i j}(p, q) \ln (j-i) \rightarrow r, \quad \text { as } i, j \rightarrow \infty,
$$

where throughout $r \geq 0$ and $i<j$.

$\left\{\xi_{n}: n \geq 1\right\}$ is called weakly dependent for $r=0$ and strongly dependent for $r>0$. In the paper, a very natural and mild assumption is

$$
\begin{aligned}
& \left|r_{i j}(p)-\frac{r}{\ln (j-i)}\right| \ln (j-i)\left(\ln D_{j-i}\right)^{1+\varepsilon}=O(1), \\
& \left|r_{i j}(p, q)-\frac{r}{\ln (j-i)}\right| \ln (j-i)\left(\ln D_{j-i}\right)^{1+\varepsilon}=O(1),
\end{aligned}
$$

where

$$
d_{k}=\frac{\exp \left(\ln ^{\alpha} k\right)}{k}, \quad D_{n}=\sum_{k=1}^{n} d_{k}, \quad \text { for } 0 \leq \alpha<\frac{1}{2} .
$$

Let $\boldsymbol{\eta}_{i}=\boldsymbol{\xi}_{i}+\mathbf{m}_{i}$ where $\mathbf{m}_{i}=\left(m_{i}, m_{i}, \ldots, m_{i}\right)$ is a real vector. The constant $m_{i}$ satisfies

$$
\beta_{n} \triangleq \max _{1 \leq i \leq n}\left|m_{i}\right|=o\left((\ln n)^{\frac{1}{2}}\right), \quad \text { as } n \rightarrow \infty
$$


$m_{n}^{*}$ is defined so that $\left|m_{n}^{*}\right| \leq \beta_{n}$ and

$$
\frac{1}{n} \sum_{i=1}^{n} \exp \left(a_{n}^{*}\left(m_{i}-m_{i}^{*}\right)-\frac{1}{2\left(m_{i}-m_{n}^{*}\right)^{2}}\right) \rightarrow 1, \quad \text { as } n \rightarrow \infty,
$$

where $a_{n}^{*}=a_{n}-\ln \ln \frac{n}{2 a_{n}}$.

\section{Results and proofs}

We mainly consider the ASCLT of the maximum of nonstationary Gaussian vector sequence satisfying (1.4), which is crucial to consider other versions of the ASCLT such as that of the maximum of stationary strongly dependent sequence and the function of the maximum. In the sequel, $a_{n} \ll b_{n}$ denotes the existence of a constant $c>0$ such that $a_{n} \ll$ $c b_{n}$ for sufficiently large $n$. We also define the normalized real vector $\mathbf{a}_{k}=\left(a_{k}, a_{k}, \ldots, a_{k}\right)$, $\mathbf{b}_{k}=\left(b_{k}, b_{k}, \ldots, b_{k}\right)$, where $a_{k}$ and $b_{k}$ are defined by (1.1). The main results are as follows.

Theorem 1 Let $\left\{\boldsymbol{\eta}_{i}: i \geq 1\right\}$ be defined by $\boldsymbol{\eta}_{i}=\boldsymbol{\xi}_{i}+\mathbf{m}_{i}$ where $\left\{\boldsymbol{\xi}_{i}: i \geq 1\right\}$ is the standard nonstationary Gaussian vector sequence with covariances satisfying (1.4). Suppose that $\left\{m_{i}\right\}$ and $m_{n}^{*}$ satisfy (1.6) and (1.7), respectively. Then

$$
\begin{gathered}
\lim _{n \rightarrow \infty} \frac{1}{D_{n}} \sum_{k=1}^{n} d_{k} I\left(\mathbf{a}_{k}\left(\max _{1 \leq i \leq k} \boldsymbol{\eta}_{i}-\mathbf{b}_{k}-\mathbf{m}_{k}^{*}\right) \leq \mathbf{x}\right) \\
=\prod_{p=1}^{d} \int_{\mathbb{R}} \exp \left(-\mathrm{e}^{-x(p)-r+\sqrt{2 r} z}\right) \mathrm{d} \Phi(z) \quad \text { a.s. }
\end{gathered}
$$

for $\mathbf{m}_{k}^{*}=\left(m_{k}^{*}, m_{k}^{*}, \ldots, m_{k}^{*}\right)$ and $\mathbf{x}=(x(1), x(2), \ldots, x(d)) \in \mathbb{R}^{d}$, where $\Phi(z)$ denotes the distribution function of a standard normal random variable.

Theorem 2 Let $\left\{\xi_{i}: i \geq 1\right\}$ is the standard nonstationary Gaussian vector sequence with covariances satisfying (1.4), we have

$$
\begin{gathered}
\lim _{n \rightarrow \infty} \frac{1}{D_{n}} \sum_{k=1}^{n} d_{k} I\left(\mathbf{a}_{k}\left(\max _{1 \leq i \leq t_{k}} \xi_{i}-\mathbf{b}_{k}\right) \leq \mathbf{x}\right) \\
=\prod_{p=1}^{d} \int_{\mathbb{R}} \exp \left(-t \mathrm{e}^{-x(p)-r+\sqrt{2 r} z}\right) \mathrm{d} \Phi(z) \quad \text { a.s., }
\end{gathered}
$$

for $\mathbf{x}=(x(1), x(2), \ldots, x(d)) \in \mathbb{R}^{d}$, where $t_{n}$ is an increasing sequence of positive integers such that $\lim _{n \rightarrow \infty} \frac{t_{n}}{n}=t(t>0)$.

In the terminology of summation procedures, we have the following corollary.

Corollary 1 Equations (2.1) and (2.2) remain valid if we replace the weight sequence $\left\{d_{k}\right.$ : $k \geq 1\}$ by $\left\{d_{k}^{*}: k \geq 1\right\}$ such that $0 \leq d_{k}^{*} \leq d_{k}, \sum_{k=1}^{\infty} d_{k}^{*}=\infty$.

Remark 1 Our results give substantial improvements for the weight sequence in Theorem A. 
Remark 2 If $\left\{\xi_{i}: i \geq 1\right\}$ is a standardized stationary Gaussian sequence, $t=1$ and $\alpha=0$, then (2.2) becomes (1.2). Thus Theorem A is a special case of Theorem 2.

Remark 3 Essentially, the problem whether Theorem 1 holds also for some $1 / 2 \leq \alpha<1$ remains open.

The following lemmas play important roles in the proofs of our theorems. The proofs are given in the Appendix.

Lemma 1 Let $\left\{\xi_{n}: n \geq 1\right\}$ and $\left\{\xi_{n}^{\prime}: n \geq 1\right\}$ be two d-dimensional independent standardized nonstationary Gaussian sequences with

$$
r_{i j}^{0}(p)=\operatorname{Cov}\left(\xi_{i}(p), \xi_{j}(p)\right), \quad r_{i j}^{0}(p, q)=\operatorname{Cov}\left(\xi_{i}(p), \xi_{j}(q)\right)
$$

and

$$
r_{i j}^{\prime}(p)=\operatorname{Cov}\left(\xi_{i}^{\prime}(p), \xi_{j}^{\prime}(p)\right), \quad r_{i j}^{\prime}(p, q)=\operatorname{Cov}\left(\xi_{i}^{\prime}(p), \xi_{j}^{\prime}(q)\right) .
$$

Write

$$
\begin{aligned}
& \rho_{i j}(p)=\max \left(\left|r_{i j}^{0}(p)\right|,\left|r_{i j}^{\prime}(p)\right|\right), \\
& \rho_{i j}(p, q)=\max \left(\left|r_{i j}^{0}(p, q)\right|,\left|r_{i j}^{\prime}(p, q)\right|\right) .
\end{aligned}
$$

Assume that (1.4) holds. Let $\mathbf{u}_{n i}=\left(u_{n i}(1), u_{n i}(2), \ldots, u_{n i}(d)\right)$ for $i \geq 1$ be real vectors such that $n\left(1-\Phi\left(u_{n i}(p)\right)\right)$ is bounded where $\Phi$ is the standard normal distribution function. There exist absolute constants $K_{1}, K_{2}$, if

$$
\max _{\substack{1 \leq i<j \leq t_{n} \\ 1 \leq p \leq d}} \rho_{i j}(p)<1 \text { and } \max _{\substack{1 \leq i<j \leq t_{n} \\ 1 \leq p \neq q \leq d}} \rho_{i j}(p, q)<1, \quad \text { for } t>0,
$$

then

$$
\begin{aligned}
\left|\mathbb{P}\left(\boldsymbol{\xi}_{j} \leq \mathbf{u}_{n j}, j=1,2, \ldots, t_{n}\right)-\mathbb{P}\left(\boldsymbol{\xi}_{j}^{\prime} \leq \mathbf{u}_{n j}, j=1,2, \ldots, t_{n}\right)\right| \\
\leq K_{1} \sum_{p=1}^{d} \sum_{1 \leq i<j \leq t_{n}}\left|r_{i j}^{0}(p)-r_{i j}^{\prime}(p)\right| \exp \left(-\frac{u_{n i}^{2}(p)+u_{n j}^{2}(p)}{2\left(1+\rho_{i j}(p)\right)}\right) \\
\quad+K_{2} \sum_{1 \leq p \neq q \leq d} \sum_{1 \leq i<j \leq t_{n}}\left|r_{i j}^{0}(p, q)-r_{i j}^{\prime}(p, q)\right| \exp \left(-\frac{u_{n i}^{2}(p)+u_{n j}^{2}(q)}{2\left(1+\rho_{i j}(p, q)\right)}\right),
\end{aligned}
$$

where $t_{n}$ is an increasing sequence of positive integers such that $\lim _{n \rightarrow \infty} \frac{t_{n}}{n}=t(t>0)$.

Lemma 2 Let $\left\{\xi_{n}: n \geq 1\right\}$ be a standardized nonstationary Gaussian vector sequence such that conditions (1.4) holds, and further suppose that $n\left(1-\Phi\left(u_{n i}(p)\right)\right)$ is bounded for $p=1,2, \ldots, d$ and $\max _{p \neq q}\left(\sup _{n \geq 0}\left|r_{n}(p, q)\right|\right)<1$. Let $\rho_{n}=\frac{r}{\ln n}, r$ defined in (1.3), $\omega_{i j}=$ 
$\max \left\{\left|r_{i j}(p)\right|, \rho_{n}\right\}, \omega_{i j}^{\prime}=\max \left\{\left|r_{i j}(p, q)\right|, \rho_{n}\right\}$. For some $\varepsilon>0$, then

$$
\sum_{p=1}^{d} \sum_{1 \leq i<j \leq t_{n}}\left|r_{i j}(p)-\rho_{n}\right| \exp \left(-\frac{u_{n i}^{2}(p)}{2\left(1+\left|\omega_{i j}\right|\right)}\right) \ll\left(\ln D_{n}\right)^{-(1+\varepsilon)}
$$

and

$$
\sum_{1 \leq p \neq q \leq d} \sum_{1 \leq i<j \leq t_{n}}\left|r_{i j}(p, q)-\rho_{n}\right| \exp \left(-\frac{u_{n i}^{2}(p)+u_{n j}^{2}(q)}{2\left(1+\left|\omega_{i j}^{\prime}\right|\right)}\right) \ll\left(\ln D_{n}\right)^{-(1+\varepsilon)}
$$

where $t_{n}$ is an increasing sequence of positive integers such that $\lim _{n \rightarrow \infty} \frac{t_{n}}{n}=t(t>0)$.

Lemma 3 Let $\left\{\tilde{\boldsymbol{\xi}}_{n}: n \geq 1\right\}$ be a standard nonstationary Gaussian vector sequence with constant covariance $\rho_{n}(p)=n / \ln n$ for $p=1,2, \ldots, d$ and $\left\{\boldsymbol{\xi}_{n}: n \geq 1\right\}$ satisfy the conditions of Theorem 1. Assume $n\left(1-\Phi\left(u_{n i}(p)\right)\right)$ is bounded for $p=1,2, \ldots, d$ and (1.4) is satisfied. For $p=1,2, \ldots, d$, then

$$
\begin{aligned}
& \left|\mathbb{E}\left(I\left(\tilde{\xi}_{1}(p) \leq u_{n 1}(p), \ldots, \tilde{\xi}_{n}(p) \leq u_{n n}(p)\right)-I\left(\xi_{1}(p) \leq u_{n 1}(p), \ldots, \xi_{n}(p) \leq u_{n n}(p)\right)\right)\right| \\
& \quad \ll\left(\ln D_{n}\right)^{-(1+\varepsilon)}, \quad \text { for some } \varepsilon>0 .
\end{aligned}
$$

Lemma 4 Let $\left\{\boldsymbol{\xi}_{n}: n \geq 1\right\}$ be a standardized nonstationary Gaussiand-dimensional vector sequence with covariances satisfying (1.4). Suppose that the assumptions of Lemma 1 hold, then

$$
\lim _{n \rightarrow \infty} \mathbb{P}\left(\mathbf{a}_{n}\left(\max _{1 \leq i \leq n} \boldsymbol{\eta}_{i}-\mathbf{b}_{n}-\mathbf{m}_{n}^{*}\right) \leq \mathbf{x}\right)=\prod_{p=1}^{d} \int_{\mathbb{R}} \exp \left(-\mathrm{e}^{-x(p)-r+\sqrt{2 r} z}\right) \mathrm{d} \Phi(z),
$$

where $\mathbf{x}=(x(1), x(2), \ldots, x(d)) \in \mathbb{R}^{d}$.

Lemma 5 Let $\zeta_{1}, \zeta_{2}, \ldots, \zeta_{n}, \ldots$, be a sequence of bounded random variables. If

$$
\operatorname{Var}\left(\sum_{k=1}^{n} d_{k} \zeta_{k}\right)=O\left(\frac{D_{n}^{2}}{\left(\ln D_{n}\right)^{1+\varepsilon}}\right), \text { for some } \varepsilon>0
$$

then

$$
\lim _{n \rightarrow \infty} \frac{1}{D_{n}} \sum_{k=1}^{n} d_{k}\left(\zeta_{k}-\mathbb{E} \zeta_{k}\right)=0 \quad \text { a.s. }
$$

Proof of Theorem 1 By Lemma 4 and the Toeplitz lemma, note that (2.1) is equivalent to

$$
\begin{array}{r}
\lim _{n \rightarrow \infty} \frac{1}{D_{n}} \sum_{k=1}^{n} d_{k}\left(I\left(\mathbf{a}_{k}\left(\max _{1 \leq i \leq n} \boldsymbol{\eta}_{i}-\mathbf{b}_{k}-\mathbf{m}_{k}^{*}\right) \leq \mathbf{x}\right)\right. \\
\left.-\mathbb{P}\left(\mathbf{a}_{k}\left(\max _{1 \leq i \leq n} \boldsymbol{\eta}_{i}-\mathbf{b}_{k}-\mathbf{m}_{k}^{*}\right) \leq \mathbf{x}\right)\right)=0 \text { a.s. }
\end{array}
$$


Let $u_{k i}(p)=\frac{x(p)}{a_{k}}+b_{k}+m_{k}^{*}-m_{i}$, by (2.3) in [1], we have $n\left(1-\Phi\left(u_{k i}(p)\right)\right) \rightarrow \tau_{p}$ for $x(p) \in \mathbb{R}$, $0 \leq \tau_{p}<\infty$. From Lemma 5 , in order to prove (2.9), for $p=1,2, \ldots, d$, it suffices to prove

$$
\begin{aligned}
& \operatorname{Var}\left(\sum_{k=1}^{n} d_{k} I\left(\xi_{1}(p) \leq \frac{x(p)}{a_{k}}+b_{k}+m_{k}^{*}-m_{1}, \ldots, \xi_{k}(p) \leq \frac{x(p)}{a_{k}}+b_{k}+m_{k}^{*}-m_{k}\right)\right) \\
& \quad=O\left(\frac{D_{n}^{2}}{\left(\ln D_{n}\right)^{1+\varepsilon}}\right) \quad \text { for some } \varepsilon>0 .
\end{aligned}
$$

Let $\zeta, \zeta_{1}, \zeta_{2}, \ldots$ be $d$-dimensional independent standardized nonstationary Gaussian sequences, where $\zeta=(\zeta, \zeta, \ldots, \zeta),\left\{\zeta_{i}=\left(\zeta_{i}(1), \zeta_{i}(2), \ldots, \zeta_{i}(d)\right), i \geq 1\right\}$. It can be shown that $\left\{\lambda_{i}(p)=\left(1-\rho_{k}\right)^{1 / 2} \zeta_{i}(p)+\rho_{k}^{1 / 2} \zeta, i \geq 1, p=1,2, \ldots, d\right\}$ have constant covariance $\rho_{k}=r / \ln k$. For $p=1,2, \ldots, d$ using the well-known $c_{2}$-inequality, the left-hand side of (2.10) can be written as

$$
\begin{aligned}
& \operatorname{Var}\left(\sum_{k=1}^{n} d_{k} I\left(\xi_{1}(p) \leq \frac{x(p)}{a_{k}}+b_{k}+m_{k}^{*}-m_{1}, \ldots, \xi_{k}(p) \leq \frac{x(p)}{a_{k}}+b_{k}+m_{k}^{*}-m_{k}\right)\right. \\
&-\sum_{k=1}^{n} d_{k} I\left(\left(1-\rho_{k}\right)^{1 / 2} \zeta_{1}(p)+\rho_{k}^{1 / 2} \zeta \leq u_{k 1}(p), \ldots,\left(1-\rho_{k}\right)^{1 / 2} \zeta_{k}(p)+\rho_{k}^{1 / 2} \zeta \leq u_{k k}(p)\right) \\
&\left.+\sum_{k=1}^{n} d_{k} I\left(\left(1-\rho_{k}\right)^{1 / 2} \zeta_{1}(p)+\rho_{k}^{1 / 2} \zeta \leq u_{k 1}(p), \ldots,\left(1-\rho_{k}\right)^{1 / 2} \zeta_{k}(p)+\rho_{k}^{1 / 2} \zeta \leq u_{k k}(p)\right)\right) \\
& \ll \operatorname{Var}\left(\sum _ { k = 1 } ^ { n } d _ { k } I \left(\left(1-\rho_{k}\right)^{1 / 2} \zeta_{1}(p)+\rho_{k}^{1 / 2} \zeta \leq u_{k 1}(p), \ldots,\right.\right. \\
&\left.\left.\quad\left(1-\rho_{k}\right)^{1 / 2} \zeta_{k}(p)+\rho_{k}^{1 / 2} \zeta \leq u_{k k}(p)\right)\right) \\
& \quad+\operatorname{Var}\left(\sum_{k=1}^{n} d_{k} I\left(\xi_{1}(p) \leq u_{k 1}(p), \ldots, \xi_{k}(p) \leq u_{k k}(p)\right)\right. \\
&\left.\quad-\sum_{k=1}^{n} d_{k} I\left(\left(1-\rho_{k}\right)^{1 / 2} \zeta_{1}(p)+\rho_{k}^{1 / 2} \zeta \leq u_{k 1}(p), \ldots,\left(1-\rho_{k}\right)^{1 / 2} \zeta_{k}(p)+\rho_{k}^{1 / 2} \zeta \leq u_{k k}(p)\right)\right) \\
&=: L_{1}+L_{2} .
\end{aligned}
$$

We will show $L_{i} \ll \frac{D_{n}^{2}}{\left(\ln D_{n}\right)^{1+\varepsilon}}, i=1,2$. For $p=1,2, \ldots, d$, clearly

$$
\begin{aligned}
L_{1}= & \mathbb{E}\left(\sum _ { k = 1 } ^ { n } d _ { k } I \left(\zeta_{1}(p) \leq\left(1-\rho_{k}\right)^{-1 / 2}\left(u_{k 1}(p)-\rho_{k}^{1 / 2} \zeta\right), \ldots,\right.\right. \\
& \left.\left.\zeta_{k}(p) \leq\left(1-\rho_{k}\right)^{-1 / 2}\left(u_{k k}(p)-\rho_{k}^{1 / 2} \zeta\right)\right)\right) \\
& -\mathbb{P}\left(\sum _ { k = 1 } ^ { n } d _ { k } I \left(\zeta_{1}(p) \leq\left(1-\rho_{k}\right)^{-1 / 2}\left(u_{k 1}(p)-\rho_{k}^{1 / 2} \zeta\right), \ldots\right.\right. \\
& \left.\left.\zeta_{k}(p) \leq\left(1-\rho_{k}\right)^{-1 / 2}\left(u_{k k}(p)-\rho_{k}^{1 / 2} \zeta\right)\right)\right)^{2}
\end{aligned}
$$




$$
\begin{aligned}
= & \int_{\mathbb{R}} \mathbb{E}\left(\sum _ { k = 1 } ^ { n } d _ { k } I \left(\zeta_{1}(p) \leq\left(1-\rho_{k}\right)^{-1 / 2}\left(u_{k 1}(p)-\rho_{k}^{1 / 2} z\right), \ldots\right.\right. \\
& \left.\zeta_{k}(p) \leq\left(1-\rho_{k}\right)^{-1 / 2}\left(u_{k k}(p)-\rho_{k}^{1 / 2} z\right)\right) \\
& -\mathbb{P}\left(\zeta_{1}(p) \leq\left(1-\rho_{k}\right)^{-1 / 2}\left(u_{k 1}(p)-\rho_{k}^{1 / 2} z\right), \ldots\right. \\
& \left.\left.\zeta_{k}(p) \leq\left(1-\rho_{k}\right)^{-1 / 2}\left(u_{k k}(p)-\rho_{k}^{1 / 2} z\right)\right)\right)^{2} \mathrm{~d} \Phi(z)
\end{aligned}
$$

where

$$
\begin{aligned}
\eta_{k}= & I\left(\zeta_{1}(p) \leq\left(1-\rho_{k}\right)^{-1 / 2}\left(u_{k 1}(p)-\rho_{k}^{1 / 2} z\right), \ldots, \zeta_{k}(p) \leq\left(1-\rho_{k}\right)^{-1 / 2}\left(u_{k k}(p)-\rho_{k}^{1 / 2} z\right)\right) \\
& -\mathbb{P}\left(\zeta_{1}(p) \leq\left(1-\rho_{k}\right)^{-1 / 2}\left(u_{k 1}(p)-\rho_{k}^{1 / 2} z\right), \ldots,\right. \\
& \left.\zeta_{k}(p) \leq\left(1-\rho_{k}\right)^{-1 / 2}\left(u_{k k}(p)-\rho_{k}^{1 / 2} z\right)\right), \quad \text { for } p=1,2, \ldots, d .
\end{aligned}
$$

Write the expectation in (2.12) as

$$
\begin{aligned}
\mathbb{E}\left(\sum_{k=1}^{n} d_{k} \eta_{k}\right)^{2} & =\sum_{k=1}^{n} d_{k}^{2} \mathbb{E}\left|\eta_{k}\right|^{2}+2 \sum_{1 \leq k<l \leq n} d_{k} d_{l}\left|\mathbb{E}\left(\eta_{k} \eta_{l}\right)\right| \\
& =: H_{1}+H_{2} .
\end{aligned}
$$

Noting that $\left|\eta_{k}\right| \leq 1, \exp \left(\ln ^{\alpha} x\right)=\exp \left(\int_{1}^{x} \frac{\alpha(\ln u)^{\alpha-1}}{u} \mathrm{~d} u\right)$, we see that $\exp \left(\ln ^{\alpha} x\right)(\alpha<1 / 2)$ is a slowly varying function at infinity. Hence,

$$
H_{1} \leq \sum_{k=1}^{n} d_{k}^{2}=\sum_{k=1}^{n} \frac{\exp \left(2 \ln ^{\alpha} k\right)}{k^{2}} \leq \sum_{k=1}^{\infty} \frac{\exp \left(2 \ln ^{\alpha} k\right)}{k^{2}}<\infty .
$$

For $H_{2}$, similarly to the proof of the main result in [1], we have

$$
\begin{aligned}
H_{2} \ll & \sum_{1 \leq k<l \leq n} d_{k} d_{l}\left(\prod_{i=k+1}^{l} \Phi\left(\left(1-\rho_{l}\right)^{-1 / 2}\left(u_{l i}(p)-\rho_{l}^{1 / 2} z\right)\right)\right. \\
& \left.-\prod_{i=1}^{l} \Phi\left(\left(1-\rho_{l}\right)^{-1 / 2}\left(u_{l i}(p)-\rho_{l}^{1 / 2} z\right)\right)\right) \\
& \ll \sum_{1 \leq k<l \leq n} d_{k} d_{l} \frac{k}{l} \\
= & \sum_{\substack{1 \leq k<l \leq n \\
l \\
k \geq \ln ^{2} D_{n}}} d_{k} d_{l} \frac{k}{l}+\sum_{\substack{1 \leq k<l \leq n \\
\frac{l}{k}<\ln ^{2} D_{n}}} d_{k} d_{l} \frac{k}{l} \\
= & T_{1}+T_{2} .
\end{aligned}
$$

For $T_{1}$, we have

$$
T_{1} \leq \sum_{1 \leq k<l \leq n} \frac{d_{k} d_{l}}{\ln ^{2} D_{n}} \leq \frac{D_{n}^{2}}{\ln ^{2} D_{n}} .
$$


According to $\mathrm{Wu}[18]$, for sufficiently large $n, 0<\alpha<\frac{1}{2}$, we have

$$
D_{n} \sim \frac{1}{\alpha}\left(\ln ^{1-\alpha} n \exp \left(\ln ^{\alpha} n\right)\right), \quad \ln D_{n} \sim \ln ^{\alpha} n, \quad \exp \left(\ln ^{\alpha} n\right) \sim \frac{\alpha D_{n}}{\left(\ln D_{n}\right)^{\frac{1-\alpha}{\alpha}}}
$$

Since $\alpha<1 / 2$ implies $(1-\alpha) / \alpha>1$, letting $0<\varepsilon<(1-\alpha) / \alpha-1$, for sufficiently large $n$, we get

$$
\begin{aligned}
T_{2} & \leq \sum_{k=1}^{n} d_{k} \sum_{l=k}^{k \ln ^{2} D_{n}} \frac{\exp \left(\ln ^{\alpha} l\right)}{l} \\
& \leq \exp \left(\ln ^{\alpha} n\right) \sum_{k=1}^{n} d_{k} \sum_{l=k}^{k \ln ^{2} D_{n}} \frac{1}{l} \\
& \ll \exp \left(\ln ^{\alpha} n\right) D_{n} \ln \ln D_{n} \ll \frac{D_{n}^{2} \ln \ln D_{n}}{\left(\ln D_{n}\right)^{\frac{1-\alpha}{\alpha}}} \\
& \leq \frac{D_{n}^{2}}{\left(\ln D_{n}\right)^{1+\varepsilon}} .
\end{aligned}
$$

Combining (2.15)-(2.18), we can get

$$
H_{2} \ll \frac{D_{n}^{2}}{\left(\ln D_{n}\right)^{1+\varepsilon}}
$$

By (2.13), (2.14), and (2.19), we have

$$
L_{1} \ll \frac{D_{n}^{2}}{\left(\ln D_{n}\right)^{1+\varepsilon}} .
$$

Clearly,

$$
\begin{aligned}
L_{2}= & \operatorname{Var}\left(\sum_{k=1}^{n} d_{k} I\left(\xi_{1}(p) \leq u_{k 1}(p), \ldots, \xi_{k}(p) \leq u_{k k}(p)\right)\right. \\
& \left.-I\left(\lambda_{1}(p) \leq u_{k 1}(p), \ldots, \lambda_{k}(p) \leq u_{k k}(p)\right)\right) \\
\leq & \sum_{k=1}^{n} d_{k}^{2} \operatorname{Var}\left(I\left(\xi_{1}(p) \leq u_{k 1}(p), \ldots, \xi_{k}(p) \leq u_{k k}(p)\right)\right. \\
& \left.-I\left(\lambda_{1}(p) \leq u_{k 1}(p), \ldots, \lambda_{k}(p) \leq u_{k k}(p)\right)\right) \\
& +2 \mid \sum_{1 \leq i<j \leq n} d_{i} d_{j} \operatorname{Cov}\left(I\left(\xi_{1}(p) \leq u_{i 1}(p), \ldots, \xi_{i}(p) \leq u_{i i}(p)\right)\right. \\
& -I\left(\lambda_{1}(p) \leq u_{i 1}(p), \ldots, \lambda_{i}(p) \leq u_{i i}(p)\right), I\left(\xi_{1}(p) \leq u_{j 1}(p), \ldots, \xi_{j}(p) \leq u_{j j}(p)\right) \\
& \left.-I\left(\lambda_{1}(p) \leq u_{j 1}(p), \ldots, \lambda_{j}(p) \leq u_{j j}(p)\right)\right) \mid \\
=: & J_{1}+2 J_{2} .
\end{aligned}
$$


Similarly to (2.14), we find that $J_{1} \leq \sum_{k=1}^{\infty} d_{k}^{2}<\infty$. Note that

$$
\begin{aligned}
J_{2} \leq & \mid \sum_{1 \leq i<j \leq n} d_{i} d_{j} \operatorname{Cov}\left(I\left(\xi_{1}(p) \leq u_{i 1}(p), \ldots, \xi_{i}(p) \leq u_{i i}(p)\right)\right. \\
& -I\left(\lambda_{1}(p) \leq u_{i 1}(p), \ldots, \lambda_{i}(p) \leq u_{i i}(p)\right), I\left(\xi_{1}(p) \leq u_{j 1}(p), \ldots, \xi_{j}(p) \leq u_{j j}(p)\right) \\
& -I\left(\lambda_{1}(p) \leq u_{j 1}(p), \ldots, \lambda_{j}(p) \leq u_{j j}(p)\right)-\left(I\left(\xi_{1}(p) \leq u_{j 1}(p), \ldots, \xi_{j}(p) \leq u_{j j}(p)\right)\right. \\
& \left.\left.-I\left(\lambda_{i}(p) \leq u_{j i}(p), \ldots, \lambda_{j}(p) \leq u_{j j}(p)\right)\right)\right) \mid \\
& +\mid \sum_{1 \leq i<j \leq n} d_{i} d_{j} \operatorname{Cov}\left(I\left(\xi_{1}(p) \leq u_{i 1}(p), \ldots, \xi_{i}(p) \leq u_{i i}(p)\right)\right. \\
& -I\left(\lambda_{1}(p) \leq u_{i 1}(p), \ldots, \lambda_{i}(p) \leq u_{i i}(p)\right), I\left(\xi_{i}(p) \leq u_{j i}(p), \ldots, \xi_{j}(p) \leq u_{j j}(p)\right) \\
& \left.-I\left(\lambda_{i}(p) \leq u_{j i}(p), \ldots, \lambda_{j}(p) \leq u_{j j}(p)\right)\right) \mid \\
=: & J_{21}+J_{22} .
\end{aligned}
$$

For $J_{21}$, we can get

$$
\begin{aligned}
J_{21} \leq & \sum_{1 \leq i<j \leq n} d_{i} d_{j}\left\{\mid \operatorname{Cov}\left(I\left(\xi_{1}(p) \leq u_{i 1}(p), \ldots, \xi_{i}(p) \leq u_{i i}(p)\right)\right.\right. \\
& -I\left(\lambda_{1}(p) \leq u_{i 1}(p), \ldots, \lambda_{i}(p) \leq u_{i i}(p)\right), I\left(\xi_{1}(p) \leq u_{j 1}(p), \ldots, \xi_{j}(p) \leq u_{j j}(p)\right) \\
& \left.-I\left(\xi_{i}(p) \leq u_{j i}(p), \ldots, \xi_{j}(p) \leq u_{j j}(p)\right)\right) \mid \\
& +\mid \operatorname{Cov}\left(I\left(\xi_{1}(p) \leq u_{i 1}(p), \ldots, \xi_{i}(p) \leq u_{i i}(p)\right)\right. \\
& -I\left(\lambda_{1}(p) \leq u_{i 1}(p), \ldots, \lambda_{i}(p) \leq u_{i i}(p)\right), I\left(\lambda_{1}(p) \leq u_{j 1}(p), \ldots, \lambda_{j}(p) \leq u_{j j}(p)\right) \\
& \left.\left.-I\left(\lambda_{i}(p) \leq u_{j i}(p), \ldots, \lambda_{j}(p) \leq u_{j j}(p)\right)\right) \mid\right\} \\
\leq & 2 \sum_{1 \leq i<j \leq n} d_{i} d_{j}\left\{\mathbb{E} \mid I\left(\xi_{1}(p) \leq u_{j 1}(p), \ldots, \xi_{j}(p) \leq u_{j j}(p)\right)\right. \\
& -I\left(\xi_{i}(p) \leq u_{j i}(p), \ldots, \xi_{j}(p) \leq u_{j j}(p)\right) \mid \\
& +\mathbb{E} \mid I\left(\lambda_{1}(p) \leq u_{j 1}(p), \ldots, \lambda_{j}(p) \leq u_{j j}(p)\right) \\
& \left.-I\left(\lambda_{i}(p) \leq u_{j i}(p), \ldots, \lambda_{j}(p) \leq u_{j j}(p)\right) \mid\right\} \\
= & 2 \sum_{1 \leq i<j \leq n} d_{i} d_{j}\left\{\mathbb{P}\left(\xi_{i}(p) \leq u_{j i}(p), \ldots, \xi_{j}(p) \leq u_{j j}(p)\right)\right. \\
& -\mathbb{P}\left(\xi_{1}(p) \leq u_{j 1}(p), \ldots, \xi_{j}(p) \leq u_{j j}(p)\right)+\mathbb{P}\left(\lambda_{i}(p) \leq u_{j i}(p), \ldots, \lambda_{j}(p) \leq u_{j j}(p)\right) \\
& \left.-\mathbb{P}\left(\lambda_{1}(p) \leq u_{j 1}(p), \ldots, \lambda_{j}(p) \leq u_{j j}(p)\right)\right\} \\
\leq & 2 \sum_{1 \leq i<j \leq n} d_{i} d_{j}\left\{\mid \mathbb{P}\left(\xi_{i}(p) \leq u_{j i}(p), \ldots, \xi_{j}(p) \leq u_{j j}(p)\right)\right. \\
& -\mathbb{P}\left(\lambda_{i}(p) \leq u_{j i}(p), \ldots, \lambda_{j}(p) \leq u_{j j}(p)\right) \mid \\
& \mid \mathbb{P}\left(\xi_{1}(p) \leq u_{j 1}(p), \ldots, \xi_{j}(p) \leq u_{j j}(p)\right) \\
& \left.\mathbb{P}(p) u_{j 1}(p), \ldots, \lambda_{j}(p) \leq u_{j j}(p)\right) \mid \\
& \\
&
\end{aligned}
$$




$$
\begin{aligned}
& +2 \mid \mathbb{P}\left(\lambda_{i}(p) \leq u_{j i}(p), \ldots, \lambda_{j}(p) \leq u_{j j}(p)\right) \\
& \left.-\mathbb{P}\left(\lambda_{1}(p) \leq u_{j 1}(p), \ldots, \lambda_{j}(p) \leq u_{j j}(p)\right) \mid\right\} .
\end{aligned}
$$

By Lemma 3 and (2.17), for $\alpha>0$, we have

$$
\begin{aligned}
2 \sum_{1 \leq i<j \leq n} & d_{i} d_{j}\left\{\mid \mathbb{P}\left(\xi_{i}(p) \leq u_{j i}(p), \ldots, \xi_{j}(p) \leq u_{j j}(p)\right)\right. \\
& -\mathbb{P}\left(\lambda_{i}(p) \leq u_{j i}(p), \ldots, \lambda_{j}(p) \leq u_{j j}(p)\right) \mid \\
& \left.+\left|\mathbb{P}\left(\xi_{1}(p) \leq u_{j 1}(p), \ldots, \xi_{j}(p) \leq u_{j j}(p)\right)-\mathbb{P}\left(\lambda_{1}(p) \leq u_{j 1}(p), \ldots, \lambda_{j}(p) \leq u_{j j}(p)\right)\right|\right\} \\
& \ll \sum_{1 \leq i<j \leq n} d_{i} d_{j}\left(\ln D_{j}\right)^{-(1+\varepsilon)}=\sum_{j=1}^{n} \frac{\exp \left(\ln ^{\alpha} j\right)}{j\left(\ln D_{j}\right)^{1+\varepsilon}} \sum_{i=1}^{j} d_{i} \\
= & \sum_{j=1}^{n} \frac{\exp \left(\ln { }^{\alpha} j\right)}{j\left(\ln D_{j}\right)^{1+\varepsilon}} D_{j} \ll \sum_{j=1}^{n} \frac{\exp \left(2 \ln { }^{\alpha} j\right)(\ln j)^{1-\alpha}}{j(\ln j)^{(1+\varepsilon) \alpha}} \\
\sim & \int_{e}^{n} \frac{\exp \left(2 \ln { }^{\alpha} x\right)(\ln x)^{1-\alpha}}{(\ln x)^{\alpha+\alpha \varepsilon}} \mathrm{d} \ln x \\
= & \int_{1}^{\ln n} \exp \left(2 y^{\alpha}\right) y^{1-2 \alpha-\alpha \varepsilon} \mathrm{d} y \\
\sim & \int_{1}^{\ln n}\left(\exp \left(2 y^{\alpha}\right) y^{1-2 \alpha-\alpha \varepsilon}+\frac{2-3 \alpha-\alpha \varepsilon}{2 \alpha} \exp \left(2 y^{\alpha}\right) y^{1-3 \alpha-\alpha \varepsilon}\right) \mathrm{d} y \\
= & \left.\frac{1}{2 \alpha} \exp \left(2 y^{\alpha}\right) y^{2-3 \alpha-\alpha \varepsilon}\right|_{1} ^{\ln n} \\
& \ll \exp \left(2 \ln { }^{\alpha} n\right)(\ln n)^{2-3 \alpha-\alpha \varepsilon} \ll \frac{D_{n}^{2}}{\left(\ln D_{n}\right)^{\frac{\alpha+\alpha \varepsilon}{\alpha}}}=\frac{D_{n}^{2}}{\left(\ln D_{n}\right)^{1+\varepsilon}} .
\end{aligned}
$$

By (2.11)-(2.15), for $p=1,2, \ldots, d$, we obtain

$$
\begin{aligned}
\sum_{1 \leq i<j \leq n} d_{i} d_{j} \mid \mathbb{P}\left(\lambda_{i}(p) \leq u_{j i}(p), \ldots, \lambda_{j}(p) \leq u_{j j}(p)\right) & \\
& -\mathbb{P}\left(\lambda_{1}(p) \leq u_{j 1}(p), \ldots, \lambda_{j}(p) \leq u_{j j}(p)\right) \mid \\
= & \sum_{1 \leq i<j \leq n} d_{i} d_{j} \mid \mathbb{P}\left(\zeta_{i}(p) \leq\left(1-\rho_{j}\right)^{-1 / 2}\left(u_{j i}(p)-\rho_{j}^{1 / 2} \zeta\right), \ldots,\right. \\
& \left.\zeta_{j}(p) \leq\left(1-\rho_{j}\right)^{-1 / 2}\left(u_{j j}(p)-\rho_{j}^{1 / 2} \zeta\right)\right) \\
& -\mathbb{P}\left(\zeta_{1}(p) \leq\left(1-\rho_{j}\right)^{-1 / 2}\left(u_{j 1}(p)-\rho_{j}^{1 / 2} \zeta\right), \ldots,\right. \\
& \left.\zeta_{j}(p) \leq\left(1-\rho_{j}\right)^{-1 / 2}\left(u_{j j}(p)-\rho_{j}^{1 / 2} \zeta\right)\right) \mid \\
= & \sum_{1 \leq i<j \leq n} d_{i} d_{j} \int_{\mathbb{R}}\left(\mathbb { P } \left(\zeta_{i}(p) \leq\left(1-\rho_{j}\right)^{-1 / 2}\left(u_{j i}(p)-\rho_{j}^{1 / 2} z\right), \ldots,\right.\right. \\
& \left.\zeta_{j}(p) \leq\left(1-\rho_{j}\right)^{-1 / 2}\left(u_{j j}(p)-\rho_{j}^{1 / 2} z\right)\right) \\
& -\mathbb{P}\left(\zeta_{1}(p) \leq\left(1-\rho_{j}\right)^{-1 / 2}\left(u_{j 1}(p)-\rho_{j}^{1 / 2} z\right), \ldots,\right. \\
& \left.\left.\zeta_{j}(p) \leq\left(1-\rho_{j}\right)^{-1 / 2}\left(u_{j j}(p)-\rho_{j}^{1 / 2} z\right)\right)\right) \mathrm{d} \Phi(z)
\end{aligned}
$$




$$
\begin{aligned}
= & \sum_{1 \leq i<j \leq n} d_{i} d_{j}\left(\int _ { \mathbb { R } } \left(\Phi^{j-i}\left(\left(1-\rho_{j}\right)^{-1 / 2}\left(\mathbf{u}_{j i}(p)-\rho_{j}^{1 / 2} z\right)\right)\right.\right. \\
& \left.\left.-\Phi^{j}\left(\left(1-\rho_{j}\right)^{-1 / 2}\left(\mathbf{u}_{j 1}(p)-\rho_{j}^{1 / 2} z\right)\right)\right) \mathrm{d} \Phi(z)\right) \\
\ll & \sum_{1 \leq i<j \leq n} d_{i} d_{j} \int_{\mathbb{R}} \frac{i}{j} \mathrm{~d} \Phi(z)=\sum_{1 \leq i<j \leq n} d_{i} d_{j} \frac{i}{j} \\
= & \frac{D_{n}^{2}}{\left(\ln D_{n}\right)^{1+\varepsilon}} .
\end{aligned}
$$

By (2.23)-(2.25), we have

$$
J_{21} \ll \frac{D_{n}^{2}}{\left(\ln D_{n}\right)^{1+\varepsilon}} .
$$

For $J_{22}$, noting that $\left\{\xi_{i}(p): i \geq 1\right\}$ and $\left\{\lambda_{i}(p): i \geq 1\right\}$ are independent, by Lemma 3 and (2.24), we get

$$
\begin{aligned}
& J_{22}=\mid \sum_{1 \leq i<j \leq n} d_{i} d_{j}\left\{\operatorname { C o v } \left(I\left(\xi_{1}(p) \leq u_{i 1}(p), \ldots, \xi_{i}(p) \leq u_{i i}(p)\right),\right.\right. \\
& \left.I\left(\xi_{i}(p) \leq u_{j i}(p), \ldots, \xi_{j}(p) \leq u_{j j}(p)\right)\right) \\
& +\operatorname{Cov}\left(I\left(\lambda_{1}(p) \leq u_{i 1}(p), \ldots, \lambda_{i}(p) \leq u_{i i}(p)\right),\right. \\
& \left.\left.I\left(\lambda_{i}(p) \leq u_{j i}(p), \ldots, \lambda_{j}(p) \leq u_{j j}(p)\right)\right)\right\} \mid \\
& =\mid \sum_{1 \leq i<j \leq n} d_{i} d_{j}\left\{\mathbb{P}\left(\xi_{1}(p) \leq u_{i 1}(p), \ldots, \xi_{i}(p) \leq u_{i i}(p), \xi_{i}(p) \leq u_{j i}(p), \ldots, \xi_{j}(p) \leq u_{j j}(p)\right)\right. \\
& -\mathbb{P}\left(\lambda_{1}(p) \leq u_{i 1}(p), \ldots, \lambda_{i}(p) \leq u_{i i}(p), \lambda_{i}(p) \leq u_{j i}(p), \ldots, \lambda_{j}(p) \leq u_{j j}(p)\right) \\
& -\mathbb{P}\left(\xi_{1}(p) \leq u_{i 1}(p), \ldots, \xi_{i}(p) \leq u_{i i}(p)\right) \mathbb{P}\left(\xi_{i}(p) \leq u_{j i}(p), \ldots, \xi_{j}(p) \leq u_{j j}(p)\right) \\
& \left.-\mathbb{P}\left(\lambda_{1}(p) \leq u_{i 1}(p), \ldots, \lambda_{i}(p) \leq u_{i i}(p)\right) \mathbb{P}\left(\lambda_{i}(p) \leq u_{j i}(p), \ldots, \lambda_{j}(p) \leq u_{j j}(p)\right)\right\} \mid \\
& \leq \sum_{1 \leq i<j \leq n} d_{i} d_{j}\left\{\mid \mathbb{P}\left(\xi_{1}(p) \leq u_{i 1}(p), \ldots, \xi_{i}(p) \leq u_{i i}(p), \xi_{i}(p) \leq u_{j i}(p), \ldots, \xi_{j}(p) \leq u_{j j}(p)\right)\right. \\
& -\mathbb{P}\left(\lambda_{1}(p) \leq u_{i 1}(p), \ldots, \lambda_{i}(p) \leq u_{i i}(p), \lambda_{i}(p) \leq u_{j i}(p), \ldots, \lambda_{j}(p) \leq u_{j j}(p)\right) \mid \\
& +\left|\mathbb{P}\left(\xi_{1}(p) \leq u_{i 1}(p), \ldots, \xi_{i}(p) \leq u_{i i}(p)\right)-\mathbb{P}\left(\lambda_{1}(p) \leq u_{i 1}(p), \ldots, \lambda_{i}(p) \leq u_{i i}(p)\right)\right| \\
& \left.+\left|\mathbb{P}\left(\xi_{i}(p) \leq u_{j i}(p), \ldots, \xi_{j}(p) \leq u_{j j}(p)\right)-\mathbb{P}\left(\lambda_{i}(p) \leq u_{j i}(p), \ldots, \lambda_{j}(p) \leq u_{j j}(p)\right)\right|\right\} \\
& +2 \mid \sum_{1 \leq i<j \leq n} d_{i} d_{j} \operatorname{Cov}\left(I\left(\lambda_{1}(p) \leq u_{i 1}(p), \ldots, \lambda_{i}(p) \leq u_{i i}(p)\right),\right. \\
& \left.I\left(\lambda_{i}(p) \leq u_{j i}(p), \ldots, \lambda_{j}(p) \leq u_{j j}(p)\right)\right) \\
& \ll \sum_{1 \leq i<j \leq n} d_{i} d_{j}\left(\ln D_{j}\right)^{-(1+\varepsilon)} \\
& +\mid \sum_{1 \leq i<j \leq n} d_{i} d_{j} \operatorname{Cov}\left(I\left(\lambda_{1}(p) \leq u_{i 1}(p), \ldots, \lambda_{i}(p) \leq u_{i i}(p)\right),\right.
\end{aligned}
$$




$$
\begin{aligned}
& \left.I\left(\lambda_{i}(p) \leq u_{j i}(p), \ldots, \lambda_{j}(p) \leq u_{j j}(p)\right)\right) \mid \\
\ll & \frac{D_{n}^{2}}{\left(\ln D_{n}\right)^{1+\varepsilon}}+\mid \sum_{1 \leq i<j \leq n} d_{i} d_{j} \operatorname{Cov}\left(I\left(\lambda_{1}(p) \leq u_{i 1}(p), \ldots, \lambda_{i}(p) \leq u_{i i}(p)\right),\right. \\
& \left.I\left(\lambda_{i}(p) \leq u_{j i}(p), \ldots, \lambda_{j}(p) \leq u_{j j}(p)\right)\right) \mid .
\end{aligned}
$$

By (2.25), we have

$$
\begin{aligned}
& \mid \sum_{1 \leq i<j \leq n} d_{i} d_{j} \operatorname{Cov}\left(I\left(\lambda_{1}(p) \leq u_{i 1}(p), \ldots, \lambda_{i}(p) \leq u_{i i}(p)\right),\right. \\
& \left.I\left(\lambda_{i}(p) \leq u_{j i}(p), \ldots, \lambda_{j}(p) \leq u_{j j}(p)\right)\right) \\
& =\mid \sum_{1 \leq i<j \leq n} d_{i} d_{j}\left\{\operatorname { C o v } \left(I\left(\lambda_{1}(p) \leq u_{i 1}(p), \ldots, \lambda_{i}(p) \leq u_{i i}(p)\right),\right.\right. \\
& \left.I\left(\lambda_{i}(p) \leq u_{j i}(p), \ldots, \lambda_{j}(p) \leq u_{j j}(p)\right)-I\left(\lambda_{1}(p) \leq u_{j 1}(p), \ldots, \lambda_{j}(p) \leq u_{j j}(p)\right)\right) \\
& +\operatorname{Cov}\left(I\left(\lambda_{1}(p) \leq u_{i 1}(p), \ldots, \lambda_{i}(p) \leq u_{i i}(p)\right),\right. \\
& \left.\left.I\left(\lambda_{1}(p) \leq u_{j 1}(p), \ldots, \lambda_{j}(p) \leq u_{j j}(p)\right)\right)\right\} \\
& \leq \sum_{1 \leq i<j \leq n} d_{i} d_{j} \mathbb{E} \mid I\left(\lambda_{1}(p) \leq u_{j 1}(p), \ldots, \lambda_{j}(p) \leq u_{j j}(p)\right) \\
& -I\left(\lambda_{i}(p) \leq u_{j i}(p), \ldots, \lambda_{j}(p) \leq u_{j j}(p)\right) \mid \\
& +\mid \sum_{1 \leq i<j \leq n} d_{i} d_{j} \operatorname{Cov}\left(I\left(\lambda_{1}(p) \leq u_{i 1}(p), \ldots, \lambda_{i}(p) \leq u_{i i}(p)\right),\right. \\
& \left.I\left(\lambda_{1}(p) \leq u_{j 1}(p), \ldots, \lambda_{j}(p) \leq u_{j j}(p)\right)\right) \\
& \leq \sum_{1 \leq i<j \leq n} d_{i} d_{j}\left(\mathbb{P}\left(\lambda_{i}(p) \leq u_{j i}(p), \ldots, \lambda_{j}(p) \leq u_{j j}(p)\right)\right. \\
& \left.-\mathbb{P}\left(\lambda_{1}(p) \leq u_{j 1}(p), \ldots, \lambda_{j}(p) \leq u_{j j}(p)\right)\right) \\
& +\operatorname{Var}\left(\sum_{i=1}^{n} d_{i} I\left(\lambda_{1}(p) \leq u_{i 1}(p), \ldots, \lambda_{i}(p) \leq u_{i i}(p)\right)\right) \\
& +\sum_{i=1}^{n} d_{i}^{2} \operatorname{Var}\left(I\left(\lambda_{1}(p) \leq u_{i 1}(p), \ldots, \lambda_{i}(p) \leq u_{i i}(p)\right)\right) \\
& \ll \frac{D_{n}^{2}}{\left(\ln D_{n}\right)^{1+\varepsilon}}+\operatorname{Var}\left(\sum_{i=1}^{n} d_{i} I\left(\lambda_{1}(p) \leq u_{i 1}(p), \ldots, \lambda_{i}(p) \leq u_{i i}(p)\right)\right) .
\end{aligned}
$$

By (2.12)-(2.20), we have

$$
\operatorname{Var}\left(\sum_{i=1}^{n} d_{i} I\left(\lambda_{1}(p) \leq u_{i 1}(p), \ldots, \lambda_{i}(p) \leq u_{i i}(p)\right)\right) \ll \frac{D_{n}^{2}}{\left(\ln D_{n}\right)^{1+\varepsilon}}
$$


Together with (2.28) and (2.29), we obtain

$$
\begin{gathered}
\mid \sum_{1 \leq i<j \leq n} d_{i} d_{j} \operatorname{Cov}\left(I\left(\lambda_{1}(p) \leq u_{i 1}(p), \ldots, \lambda_{i}(p) \leq u_{i i}(p)\right),\right. \\
\left.\quad I\left(\lambda_{i}(p) \leq u_{j i}(p), \ldots, \lambda_{j}(p) \leq u_{j j}(p)\right)\right) \mid \ll \frac{D_{n}^{2}}{\left(\ln D_{n}\right)^{1+\varepsilon}} .
\end{gathered}
$$

Hence by (2.27) and (2.30), we have

$$
J_{22} \ll \frac{D_{n}^{2}}{\left(\ln D_{n}\right)^{1+\varepsilon}} .
$$

By (2.21), (2.22), (2.26), and (2.31)), for $\alpha>0$, we get

$$
L_{2} \ll \frac{D_{n}^{2}}{\left(\ln D_{n}\right)^{1+\varepsilon}} .
$$

Thus (2.10)-(2.32) together establish (2.9). The proof is completed.

Proof of Theorem 2 According to Lin et al. [1], we have

$$
\begin{aligned}
\lim _{n \rightarrow \infty} \mathbb{P}\left(a_{k}\left(\max _{1 \leq i \leq t_{k}} \xi_{i}(p)-b_{k}\right) \leq \mathbf{x}\right) \\
\quad=\int_{\mathbb{R}} \exp \left(-t \mathrm{e}^{-x(p)-r+\sqrt{2 r} z}\right) \mathrm{d} \Phi(z), \quad \text { for } p=1,2, \ldots, d .
\end{aligned}
$$

By similar methods to the ones used to prove Lemma 4, we can prove

$$
\lim _{n \rightarrow \infty} \mathbb{P}\left(\mathbf{a}_{k}\left(\max _{1 \leq i \leq t_{k}} \boldsymbol{\xi}_{i}-\mathbf{b}_{k}\right) \leq \mathbf{x}\right)=\prod_{p=1}^{d} \int_{\mathbb{R}} \exp \left(-t \mathrm{e}^{-x(p)-r+\sqrt{2 r} z}\right) \mathrm{d} \Phi(z)
$$

Note $\lim _{n \rightarrow \infty} \frac{t_{n}}{n}=t(t>0)$ and we have Lemma 2, so the remainder of the proof is similar to that of Theorem 1. We thus omit it.

\section{Appendix}

Proof of Lemma 1 See Lemma 3.1 of [17].

Proof of Lemma 2 Using Lemma 2.1 in [15], we have

$$
\begin{aligned}
& \sum_{1 \leq p \neq q \leq d} \sum_{1 \leq i<j \leq t_{n}}\left|r_{i j}(p, q)-\rho_{n}\right| \exp \left(-\frac{u_{n i}^{2}(p)+u_{n j}^{2}(q)}{2\left(1+\left|\omega_{i j}^{\prime}\right|\right)}\right) \\
& \ll \sum_{1 \leq i<j \leq t_{n}}\left|r_{i j}(p, q)-\rho_{n}\right| \exp \left(-\frac{u_{n i}^{2}(p)+u_{n j}^{2}(q)}{2\left(1+\left|\omega_{i j}^{\prime}\right|\right)}\right) \\
& \ll \frac{1}{\alpha n^{2}} \sum_{\substack{j-i>n^{\alpha} \\
1 \leq i<j \leq t_{n}}}\left|r_{i j} \ln (j-i)-r\right|+\frac{r}{n^{2}} \sum_{\substack{j-i \leq n^{\alpha} \\
1 \leq i<j \leq t_{n}}}\left|\frac{\ln n}{\ln (j-i)}-1\right|=: J_{1}+J_{2} .
\end{aligned}
$$


According to $\mathrm{Wu}$ [18], for sufficiently large $n$, we have $\ln D_{n} \sim \ln ^{\alpha} n$, for $0<\alpha<\frac{1}{2}$. Some simple calculations immediately induce

$$
J_{1} \leq \frac{1}{\alpha n^{2}} \sum_{\substack{j-i>n^{\alpha} \\ 1 \leq i<j \leq t_{n}}} \frac{1}{(\ln \ln (j-i))^{1+\varepsilon}} \ll \frac{c}{(\ln \ln n)^{1+\varepsilon}} \ll\left(\ln D_{n}\right)^{-(1+\varepsilon)}
$$

and

$$
J_{2} \leq \frac{1}{\ln n^{\alpha}} \iint_{0 \leq x<y \leq t} \ln |y-x| \mathrm{d} x \mathrm{~d} y \ll \frac{c}{(\ln \ln n)^{1+\varepsilon}} \ll\left(\ln D_{n}\right)^{-(1+\varepsilon)} .
$$

Combining (A.1), (A.2), and (A.3), we get the desired result.

Proof of Lemma 3 For $t=1$, using Lemmas 1 and 2, the proof can be obtained simply.

Proof of Lemma 4 Let $\left\{\xi_{1}^{\prime}(p), \xi_{2}^{\prime}(p), \ldots, \xi_{n}^{\prime}(p)\right\}$ have the same distribution as $\left\{\xi_{1}(p), \xi_{2}(p), \ldots\right.$, $\left.\xi_{n}(p)\right\}$, for $p=1,2, \ldots, d$, but $\left\{\xi_{1}^{\prime}(p), \xi_{2}^{\prime}(p), \ldots, \xi_{n}^{\prime}(p)\right\}$ is independent of $\left\{\xi_{1}^{\prime}(q), \xi_{2}^{\prime}(q), \ldots, \xi_{n}^{\prime}(q)\right\}$, as $p \neq q$. Denote $u_{n i}(p)=\frac{x(p)}{a_{n}}+b_{n}+m_{n}^{*}-m_{i}, \mathbf{u}_{n i}=\left(u_{n i}(1), u_{n i}(2), \ldots, u_{n i}(d)\right)$ is a real vector. By (3.2) in [19] and Lemma 1, we have

$$
\begin{aligned}
\mid \mathbb{P} & \left(\mathbf{a}_{n}\left(\max _{1 \leq i \leq n} \boldsymbol{\eta}_{i}-\mathbf{b}_{n}-\mathbf{m}_{n}^{*}\right) \leq \mathbf{x}\right)-\prod_{p=1}^{d} \mathbb{P}\left(a_{n}\left(\max _{1 \leq i \leq n} \eta_{i}(p)-b_{n}-m_{n}^{*}\right) \leq x(p)\right) \mid \\
= & \left|\mathbb{P}\left(\boldsymbol{\xi}_{i} \leq \mathbf{u}_{n i}, i=1,2, \ldots, n\right)-\mathbb{P}\left(\boldsymbol{\xi}_{i}^{\prime} \leq \mathbf{u}_{n i}, i=1,2, \ldots, n\right)\right| \\
\leq & K_{1} \sum_{p=1}^{d} \sum_{1 \leq i<j \leq n}\left|r_{i j}^{0}(p)-r_{i j}^{\prime}(p)\right| \exp \left(-\frac{u_{n i}^{2}(p)+u_{n j}^{2}(p)}{2\left(1+\rho_{i j}(p)\right)}\right) \\
& +K_{2} \sum_{1 \leq p \neq q \leq d} \sum_{1 \leq i<j \leq n}\left|r_{i j}^{0}(p, q)-r_{i j}^{\prime}(p, q)\right| \exp \left(-\frac{u_{n i}^{2}(p)+u_{n j}^{2}(q)}{2\left(1+\rho_{i j}(p, q)\right)}\right) \\
= & : A_{1}+A_{2} .
\end{aligned}
$$

$\left\{\xi_{1}^{\prime}(p), \xi_{2}^{\prime}(p), \ldots, \xi_{n}^{\prime}(p)\right\}$ has the same distribution as $\left\{\xi_{1}(p), \xi_{2}(p), \ldots, \xi_{n}(p)\right\}$, which implies $r_{i j}^{0}(p)=r_{i j}^{\prime}(p)$. Then $A_{1}=0$.

Notice that $\left\{\xi_{1}^{\prime}(p), \xi_{2}^{\prime}(p), \ldots, \xi_{n}^{\prime}(p)\right\}$ is independent of $\left\{\xi_{1}^{\prime}(q), \xi_{2}^{\prime}(q), \ldots, \xi_{n}^{\prime}(q)\right\}$, as $p \neq q$, thus $r_{i j}^{\prime}(p, q)=0$. By using Lemma 3.2 in [17], we have

$$
\begin{aligned}
A_{2} & \ll \sum_{1 \leq p \neq q \leq d} \sum_{1 \leq i<j \leq n}\left|r_{i j}^{0}(p, q)\right| \exp \left(-\frac{u_{n i}^{2}(p)+u_{n j}^{2}(q)}{2\left(1+\rho_{i j}(p, q)\right)}\right) \\
& \ll\left(\ln D_{n}\right)^{-(1+\varepsilon)} \rightarrow 0 .
\end{aligned}
$$

By (3.4),

$$
\begin{aligned}
\lim _{n \rightarrow \infty} \mathbb{P}\left(\mathbf{a}_{n}\left(\max _{1 \leq i \leq n} \boldsymbol{\eta}_{i}-\mathbf{b}_{n}-\mathbf{m}_{n}^{*}\right) \leq \mathbf{x}\right) \\
=\lim _{n \rightarrow \infty} \prod_{p=1}^{d} \mathbb{P}\left(a_{n}\left(\max _{1 \leq i \leq n} \eta_{i}(p)-b_{n}-m_{n}^{*}\right) \leq x(p)\right) .
\end{aligned}
$$


From Theorem of [16], we get

$$
\lim _{n \rightarrow \infty} \prod_{p=1}^{d} \mathbb{P}\left(a_{n}\left(\max _{1 \leq i \leq n} \eta_{i}(p)-b_{n}-m_{n}^{*}\right) \leq x(p)\right)=\prod_{p=1}^{d} \int_{\mathbb{R}} \exp \left(-\mathrm{e}^{-x(p)-r+\sqrt{2 r} z}\right) \mathrm{d} \Phi(z) .
$$

Combining (3.5) and (3.6), the proof is completed.

Proof of Lemma 5 The proof can be found in Lemma 2.2 obtained by Wu [18].

Competing interests

The authors declare that they have no competing interests.

\section{Authors' contributions}

XZ conceived of the study and drafted and completed the manuscript. QW participated in the discussion of the manuscript. XZ and QW read and approved the final manuscript.

\section{Authors' information}

Xiang Zeng, Lecturer, Master, working in the field of probability and statistics.

\section{Acknowledgements}

The authors were very grateful to the editor and two anonymous referees for their careful reading of the manuscript and valuable suggestions which helped in significantly improving an earlier version of this article. Supported by the National Natural Science Foundation of China (11361019), project supported by Program of the Guangxi China Science Foundation (2015GXNSFAA139008, 2014GXNSFAA118015, 2013GXNSFAA278003), and the Support of the Scientific Research Project of Education Department of Guangxi (YB2014150).

\section{Received: 15 January 2016 Accepted: 2 June 2016 Published online: 17 June 2016}

\section{References}

1. Lin, FM, Fu, Y, Jiang, YY: Almost sure limit theorems for the maxima of some strongly dependent Gaussian sequences. Comput. Math. Appl. 62(2), 635-640 (2011)

2. Cheng, SH, Peng, L, Qi, YC: Almost sure convergence in extreme value theory. Math. Nachr. 190(1), 43-50 (1998)

3. Fahrner, I, Stadtmüller, U: On almost sure max-limit theorems. Stat. Probab. Lett. 37(3), 229-236 (1998)

4. Berkes, I, Csáki, E: A universal result in almost sure central limit theory. Stoch. Process. Appl. 94, 105-134 (2001)

5. Wu, QY: Almost sure limit theorems for stable distribution. Stat. Probab. Lett. 81(6), 662-672 (2011)

6. Wu, QY: An almost sure central limit theorem for the weight function sequences of NA random variables. Proc. Indian Acad. Sci. Math. Sci. 121(3), 369-377 (2011)

7. Wu, QY: A note on the almost sure limit theorem for self-normalized partial sums of random variables in the domain of attraction of the normal law. J. Inequal. Appl. 2012(1), 17 (2012)

8. Wu, QY: Almost sure central limit theory for products of sums of partial sums. Appl. Math. J. Chin. Univ. Ser. B 27(2), 169-180 (2012)

9. Wu, QY: An improved result in almost sure central limit theory for products of partial sums with stable distribution. Chin. Ann. Math., Ser. B 33(6), 919-930 (2012)

10. Peng, ZX, Li, JN, Nadarajah, S: Almost sure convergence of extreme order statistics. Electron. J. Stat. 3, 546-556 (2009)

11. Peng, ZX, Tan, ZQ, Nadarajah, S: Almost sure central limit theorem for the products of U-statistics. Metrika 73(1), 61-76 (2009)

12. Peng, ZX, Wang, LL, Nadarajah, S: Almost sure central limit theorem for partial sums and maxima. Math. Nachr. 282(4), 632-636 (2009)

13. Tan, ZQ, Peng, ZX: Almost sure convergence for non-stationary random sequences. Stat. Probab. Lett. 79(7), 857-863 (2009)

14. Csáki, E, Gonchigdanzan, K: Almost sure limit theorem for the maximum of stationary Gaussian sequences. Stat. Probab. Lett. 58(2), 195-203 (2002)

15. Lin, FM: Almost sure limit theorem for the maxima of strongly dependent Gaussian sequences. Electron. Commun. Probab. 14, 224-231 (2009)

16. Leadbetter, MR, Lindgren, G, Rootzén, H: Extremes and Related Properties of Random Sequences and Processes. Springer, New York (1983)

17. Chen, ZC, Peng, ZX, Zhang, HY: An almost sure limit theorem for the maxima of multivariate stationary Gaussian sequences. J. Aust. Math. Soc. 86(3), 315-321 (2009)

18. Wu, QY, Chen, PY: An improved result in almost sure central limit theorem for self-normalized products of partial sums. J. Inequal. Appl. 2013(1), 129 (2013)

19. Zeng, X, Wu, QY: The improved results in almost sure central limit theorem for the maxima of strongly dependent stationary Gaussian vector sequences. J. Inequal. Appl. 2015, 224 (2015) 